\title{
Experience with low-energy gold-gold operations in RHIC during FY 2010
}

C. Montag, T. Satogata, L. A. Ahrens, M. Bai, J. Beebe-Wang, I. Blackler, M. Blaskiewicz, J.M. Brennan, K.A. Brown, D. Bruno, J.J. Butler, C. Carlson, R. Connolly, T. D’Ottavio, K.A. Drees, W. Fischer, W. Fu, D.M. Gassner, M. Harvey, T. Hayes, H. Huang, R.L. Hulsart, P.F. Ingrassia, A. Jain, N.A. Kling, M. Lafky, J.S. Laster, R.C. Lee, Y. Luo, W.W. MacKay, M. Mapes, G.J. Marr, A. Marusic, K. Mernick, R.J. Michnoff, M.G. Minty, J. Morris, C. Naylor, S. Nemesure, F.C. Pilat, V. Ptitsyn, G. Robert-Demolaize, T. Roser, P. Sampson, V. Schoefer, C. Schultheiss, F. Severino, T.C. Shrey, K.S. Smith, S. Tepikian,

P. Thieberger, D. Trbojevic, N. Tsoupas, J.E. Tuozzolo, M. Wilinski, A. Zaltsman, K. Zeno, S.Y. Zhang

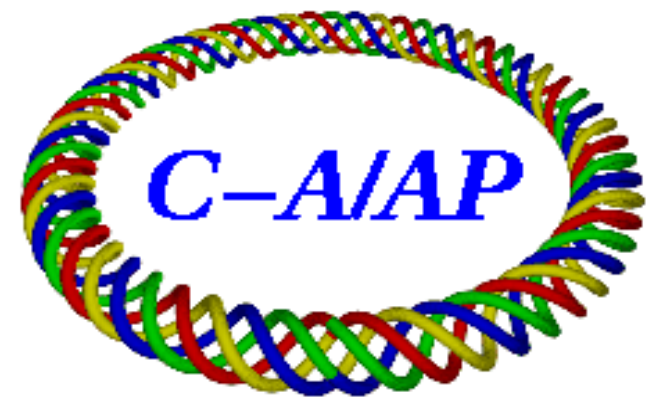

\section{Collider-Accelerator Department Brookhaven National Laboratory Upton, NY 11973}

Notice: This document has been authorized by employees of Brookhaven Science Associates, LLC under Contract No. DE-AC02-98CH10886 with the U.S. Department of En ergy. The United States Government retains a nonexclusive, paid-up, irrevocable, world-wide license to publish or reproduce the published form of this document, or allow others to do so, for United States Government purposes. 


\title{
Experience with low-energy gold-gold operations in RHIC during FY 2010
}

\author{
C. Montag, T. Satogata, L. A. Ahrens, M. Bai, J. Beebe-Wang, \\ I. Blackler, M. Blaskiewicz, J.M. Brennan, K.A. Brown, D. Bruno, \\ J.J. Butler, C. Carlson, R. Connolly, T. D'Ottavio, K.A. Drees, \\ W. Fischer, W. Fu, D.M. Gassner, M. Harvey, T. Hayes, \\ H. Huang, R.L. Hulsart, P.F. Ingrassia, A. Jain, N.A. Kling, \\ M. Lafky, J.S. Laster, R.C. Lee, Y. Luo, W.W. MacKay, \\ M. Mapes, G.J. Marr, A. Marusic, K. Mernick, R.J. Michnoff, \\ M.G. Minty, J. Morris, C. Naylor, S. Nemesure, F.C. Pilat, \\ V. Ptitsyn, G. Robert-Demolaize, T. Roser, P. Sampson, \\ V. Schoefer, C. Schultheiss, F. Severino, T.C. Shrey, K.S. Smith, \\ S. Tepikian, P. Thieberger, D. Trbojevic, N. Tsoupas, \\ J.E. Tuozzolo, M. Wilinski, A. Zaltsman, K. Zeno, S.Y. Zhang
}

October 7, 2011

\section{Introduction}

During Run-10, RHIC operated at several different $\mathrm{Au}-\mathrm{Au}$ collision energies, as requested mainly by the STAR collaboration in a quest to search for the critical point in the QGP phase diagram. The center-of-mass energies $\sqrt{s_{\mathrm{NN}}}$ are listed in Table 1 , together with the respective start and end dates and the duration of the respective run at each energy.

While STAR defines "low energy" as anything below $\sqrt{s_{\mathrm{NN}}}=39 \mathrm{GeV}$, we focus in the scope of this paper on energies below the regular RHIC injection energy of $\sqrt{s_{\mathrm{NN}}} \approx 20 \mathrm{GeV}$, since this energy regime is particularly challenging for stable RHIC operations. Figures 1 and 2 show the evolution of beam intensity and luminosity during the course of the $\sqrt{s_{\mathrm{NN}}}=7.7 \mathrm{GeV}$ and $11.5 \mathrm{GeV}$ run. In the following sections we will recapitulate the modifications during the run that led to significant performance improvements, and summarize what was learned at the various energies for possible application in future runs.

Table 1: List of center-of-mass energies $\sqrt{s_{\mathrm{NN}}}$ during the RHIC Au-Au run in FY $2010[1,2]$.

\begin{tabular}{|c|c|c|c|c|}
\hline$\sqrt{s_{\mathrm{NN}}}[\mathrm{GeV}]$ & Start date & End date & \# Days & int. Luminosity $\left[\mu \mathrm{b}^{-1}\right]$ \\
\hline \hline 200 & Dec 5, 2009 & Mar 18, 2010 & 103 & 5240 \\
62.4 & Mar 18, 2010 & Apr 9, 2010 & 22 & 281 \\
39 & Apr 9, 2010 & Apr 22, 2010 & 13 & 107 \\
7.7 & Apr 22, 2010 & May 27, 2010 & 35 & 2.48 \\
11.5 & May 27, 2010 & Jun 7, 2010 & 11 & 7.82 \\
5 (test) & Jun 7, 2010 & Jun 9, 2010 & 2.5 & 0 \\
\hline
\end{tabular}




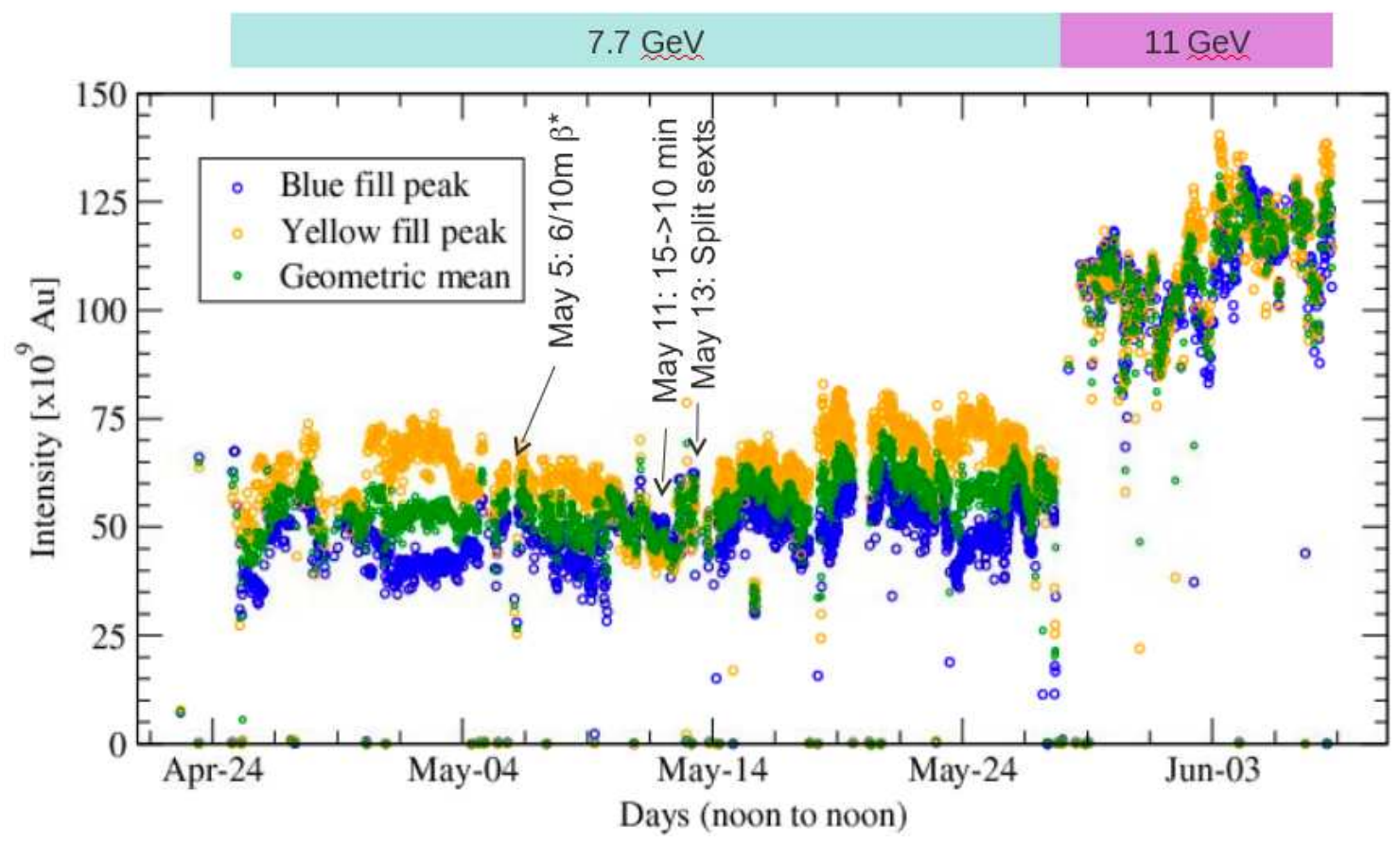

Figure 1: Injected peak intensities during the $\sqrt{s_{\mathrm{NN}}}=7.7 \mathrm{GeV}$ and $11.5 \mathrm{GeV}$ run.

\section{The $\sqrt{s_{\mathrm{NN}}}=7.7 \mathrm{GeV}$ run}

At beam energies well below the regular operating range, the velocity $v$ of the beams differs significantly from the speed of light, $c$. With the harmonic number $h$ of the $28 \mathrm{MHz} \mathrm{RF}$ system fixed at $h=360$, the required RF frequency would be outside the cavity tuning range of $f_{\mathrm{RF}}=$ $27.98 \ldots 28.17 \mathrm{MHz}$. To overcome this limitation the harmonic number was modified for low energy operations. Table 2 lists the main machine parameters for the low energy runs with the $200 \mathrm{GeV}$ parameters added for comparison.

With a revolution frequency of only $75875 \mathrm{~Hz}$ compared to the regular $\sqrt{s_{\mathrm{NN}}}=200 \mathrm{GeV}$ store value of $78193 \mathrm{~Hz}$, the harmonic number of the $28 \mathrm{MHz} \mathrm{RF}$ system was changed from its nominal value of $h=360$ to 369, resulting in an RF frequency within the tuning range of the cavities, namely $27.998 \mathrm{MHz}$. Since $h=369$ is a multiple of 9 , simultaneous collisions at both STAR and PHENIX were provided.

The $\sqrt{s_{\mathrm{NN}}}=7.7 \mathrm{GeV}$ run started in a RHIC configuration with $\beta^{*}=10 \mathrm{~m}$ at STAR and PHENIX, and a nominal store length of 20 minutes. Shortening the stores to 15 minutes on April 30 approximately doubled the average collision rate, and on May 5 the focusing at STAR was changed to $\beta^{*}=6 \mathrm{~m}$, which significantly improved the luminosity even further. A similar squeeze at PHENIX was unsuccessful since it resulted in unacceptably high background rates. On May 11 the further reduction of the nominal store length from 15 to 10 minutes increased the average luminosity once more.

During this run, it was realized that STAR background rejection capabilities for low energy operations are significantly better than those of PHENIX. The STAR data acquisition can perform background rejection based on online vertex reconstruction from the TPC, and reject background events that have reconstructed vertices outside of the beampipe or upstream of the detector. PHENIX does not have this capability and could thus not benefit from a beta squeeze due to the 


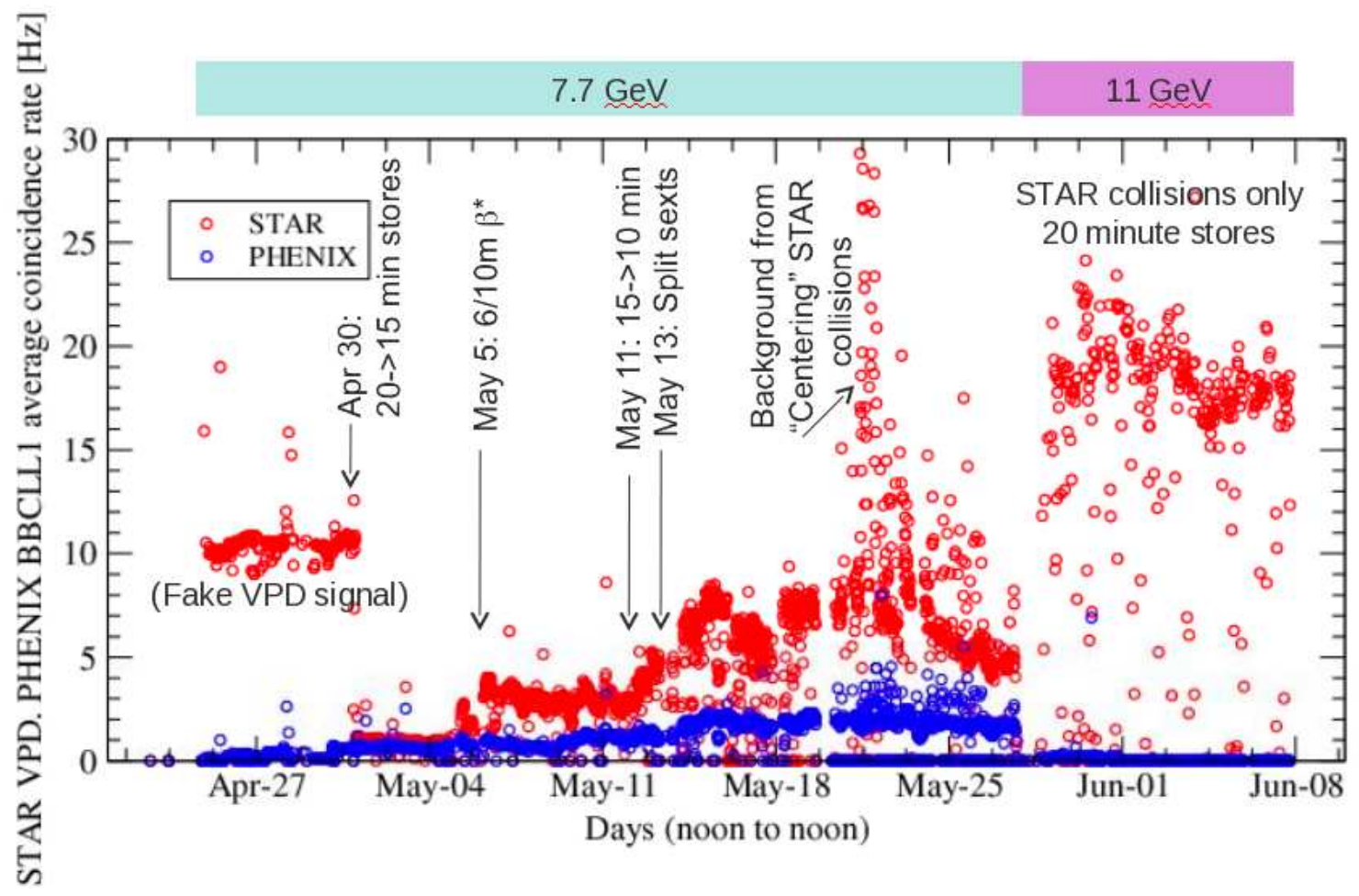

Figure 2: Average store coincidence rates at STAR and PHENIX during the $\sqrt{s_{\mathrm{NN}}}=7.7 \mathrm{GeV}$ and $11.5 \mathrm{GeV}$ run.

\begin{tabular}{|l|r|r|r|r|}
\hline$\sqrt{s_{\mathrm{NN}}}[\mathrm{GeV}]$ & 200 & 11.5 & 7.7 & 5.0 \\
$h$ & 360 & 363 & 369 & 387 \\
$\gamma$ & 108.401 & 6.175 & 4.135 & 2.685 \\
$\beta$ & 1.000 & 0.987 & 0.970 & 0.928 \\
$B \rho[\mathrm{Tm}]$ & 839.541 & 47.197 & 31.074 & 19.299 \\
$I_{\text {main dipole }}[\mathrm{A}]$ & 4887.394 & 274.759 & 180.895 & 112.348 \\
$I_{\text {main quad }}[\mathrm{A}]$ & 4548.873 & 255.728 & 168.366 & 104.567 \\
$f_{\text {rev }}[\mathrm{Hz}]$ & 78192.963 & 77164.203 & 75874.890 & 72570.182 \\
$f_{\mathrm{RF}, h=360}[\mathrm{MHz}]$ & 28.149 & 27.779 & 27.315 & 16.125 \\
$f_{\mathrm{RF}, \operatorname{modified~} h}[\mathrm{MHz}]$ & 28.149 & 28.011 & 27.998 & 28.085 \\
\hline
\end{tabular}

Table 2: RHIC machine parameters at various energies. 


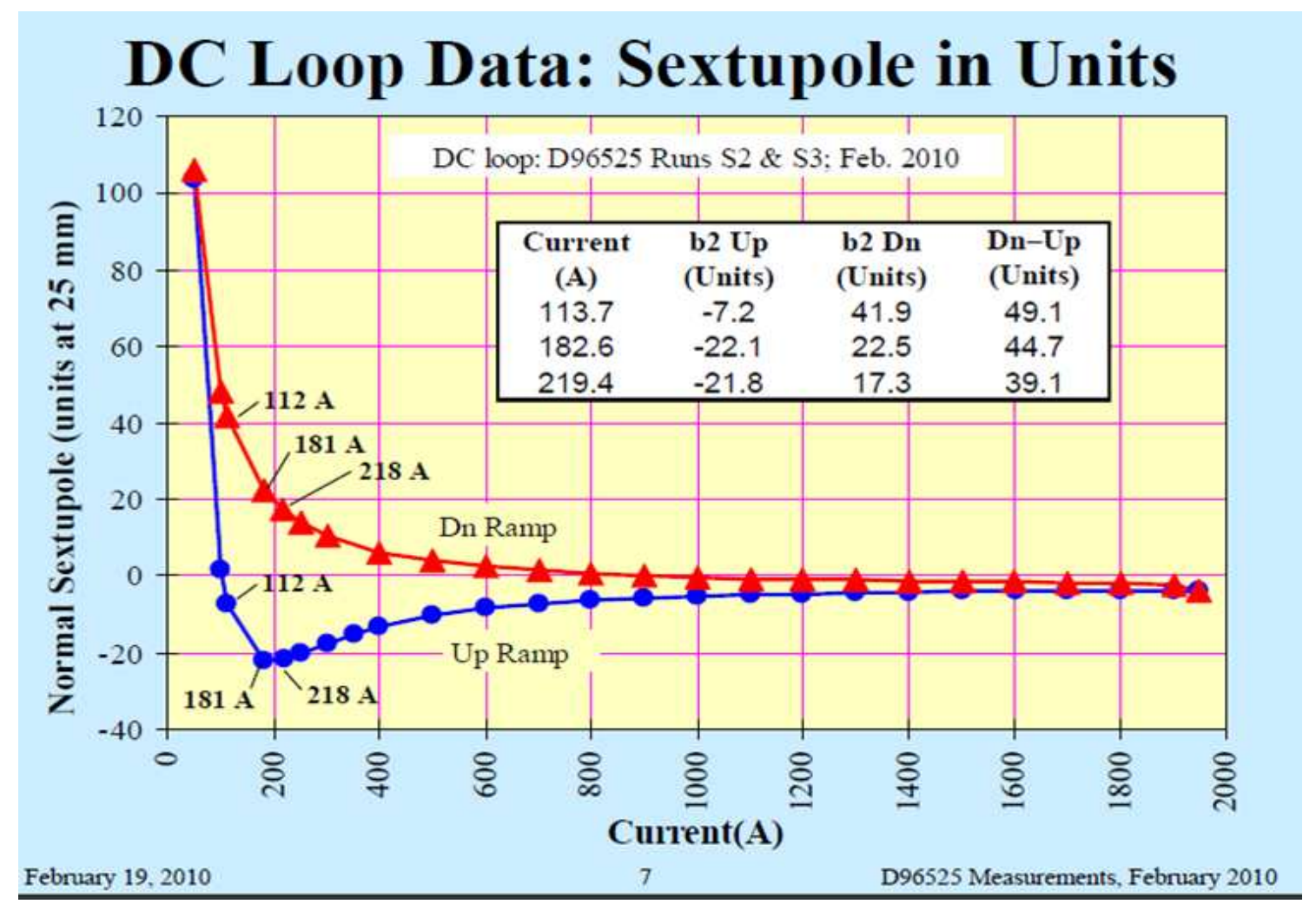

Figure 3: Measured sextupole error $b_{2}$ during the up- and down-ramp of a spare RHIC dipole.

substantial increase in their backgrounds.

Due to the expected low mutual forward neutron production rate at this energy, the ZDC detector is not suitable. The BBC detectors are plagued by high backgrounds and are therefore difficult to use as well. Therefore, the signal from the STAR VPD (vertex position detector) was made available to $\mathrm{C}-\mathrm{AD}$ to provide a cleaner and more reliable collision rate measurement. However, since the signal was only provided with some delay, a background corrected BBC signal was used for integrating the delivered luminosity.

In preparation for the low energy run, magnet multipoles had been measured on one spare dipole and quadrupole each to study the nonlinear field errors of the supcerconducting RHIC magnets at the low currents required for low energy operation. Field measurements were performed during the up- and down-ramp of a hysteresis cycle between the RHIC park current of $50 \mathrm{~A}$ and a maximum current of $2000 \mathrm{~A}$, which corresponds to a gold beam energy of $40 \mathrm{GeV} /$ nucleon. As Figure 3 shows, the sextupole error $b_{2}$ in the RHIC dipoles reaches its largest absolute value of -22 units during the up-ramp at a magnet current that corresponds to a $\mathrm{Au}$-Au collision energy of $\sqrt{s_{\mathrm{NN}}}=7.7 \mathrm{GeV}$, while the corresponding value for the down-ramp is +22 units.

Model predictions based on these measurements showed that the polarity of all the SD sextupoles had to be reversed in order to enable chromaticity correction. However, later on during the run it was realized that the vertical chromaticity could not be corrected to values below +10 units in this configuration. To overcome this limitation, a novel "split sextupole" scheme was implemented on May 13, with every other SD sextupole reverted back to its standard polarity [3]. This modification led to an increased chromaticity correction capability, and resulted in a significant reduction of beam decay (Fig. 4) and thus an increased integrated luminosity.

Without the need for hysteresis ramps, operations was very smooth, with store-to-store turnaround 


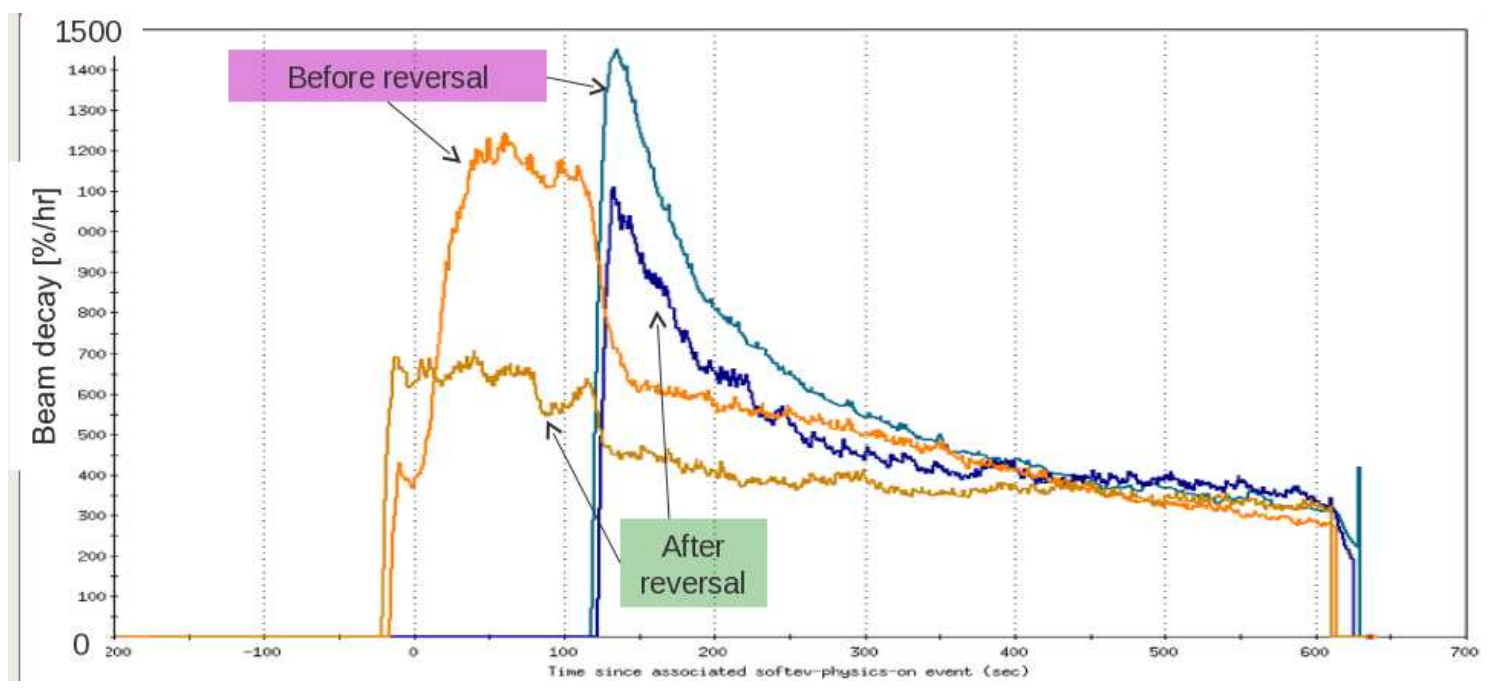

Figure 4: Beam decay rates during a typical store at $\sqrt{s_{\mathrm{NN}}}=7.7 \mathrm{GeV}$, before and after implementing the "split sextupole" scheme.

times of 2 minutes (Fig. 5). The time spent in "physics" mode was often around 80 percent. This smooth, continuous operation had the additional benefit of allowing us to study slow orbit drifts in great detail (Fig. 6).

\section{The $\sqrt{s_{\mathrm{NN}}}=11.5 \mathrm{GeV}$ run}

For the $\sqrt{s_{\mathrm{NN}}}=11.5 \mathrm{GeV}$ run the harmonic number was set to $h=363$ (see Table 2). Since this number is not a multiple of 9 collisions could be provided only at the STAR detector. Despite the miniscule total beam-beam parameter due to the single collision point, a very significant reduction in beam decay was observed as soon as the opposing beam was dumped at the end of each store (Fig. 7). While the root cause of this phenomenon is not yet understood, it is believed that strong space charge effects play a significant role. Simulation studies are currently underway in an effort to understand this phenomenon.

\section{Operating at $\sqrt{s_{\mathrm{NN}}}=5 \mathrm{GeV}$}

At the end of the FY 2010 run, a two-day test was performed at $\sqrt{s_{\mathrm{NN}}}=5 \mathrm{GeV}$, as illustrated by Figure 8. Operations was limited intentionally to the Blue ring to focus effort.

In the beginning, the "down" branch of the hysteresis cycle (see Figure 3 ) was used in an attempt to reduce the sextupole error $b_{2}$ of the RHIC dipoles. When no beam storage was achieved after a couple of hours, this attempt was abandoned, and a new approach was made using the "up" branch. With unbunched beam a peak intensity of $1.1 \cdot 10^{8}$ gold ions was achieved in this configuration, but after RF capture was attempted this dropped by almost an order of magnitude to $2 \cdot 10^{7}$ gold ions after a few turns. After about 18 hours of operation it was discovered that the "split sextupole" scheme was not reflected in the online model at this beam energy. However, no improvement was observed after this was corrected, and the final beam lifetimes (double-exponential fit) were around $4 \mathrm{sec}$ for 65 percent of the injected intensity, and $40 \mathrm{sec}$ for the remaining 35 percent.

These beam lifetimes are orders of magnitude below what would be required for successful collider operation at this energy. However, careful analysis of the measured multipole data later 


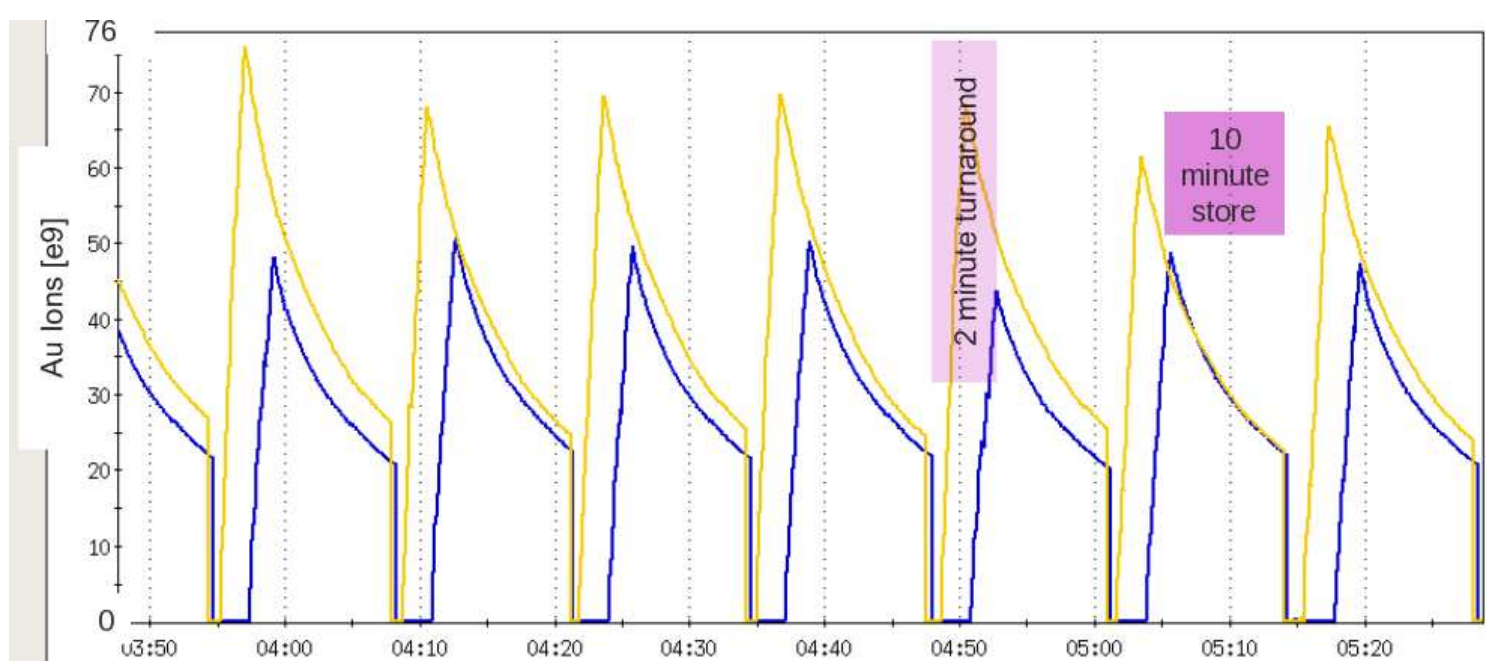

Figure 5: Beam intensities during a number of stores during the $\sqrt{s_{\mathrm{NN}}}=7.7 \mathrm{GeV}$ run.

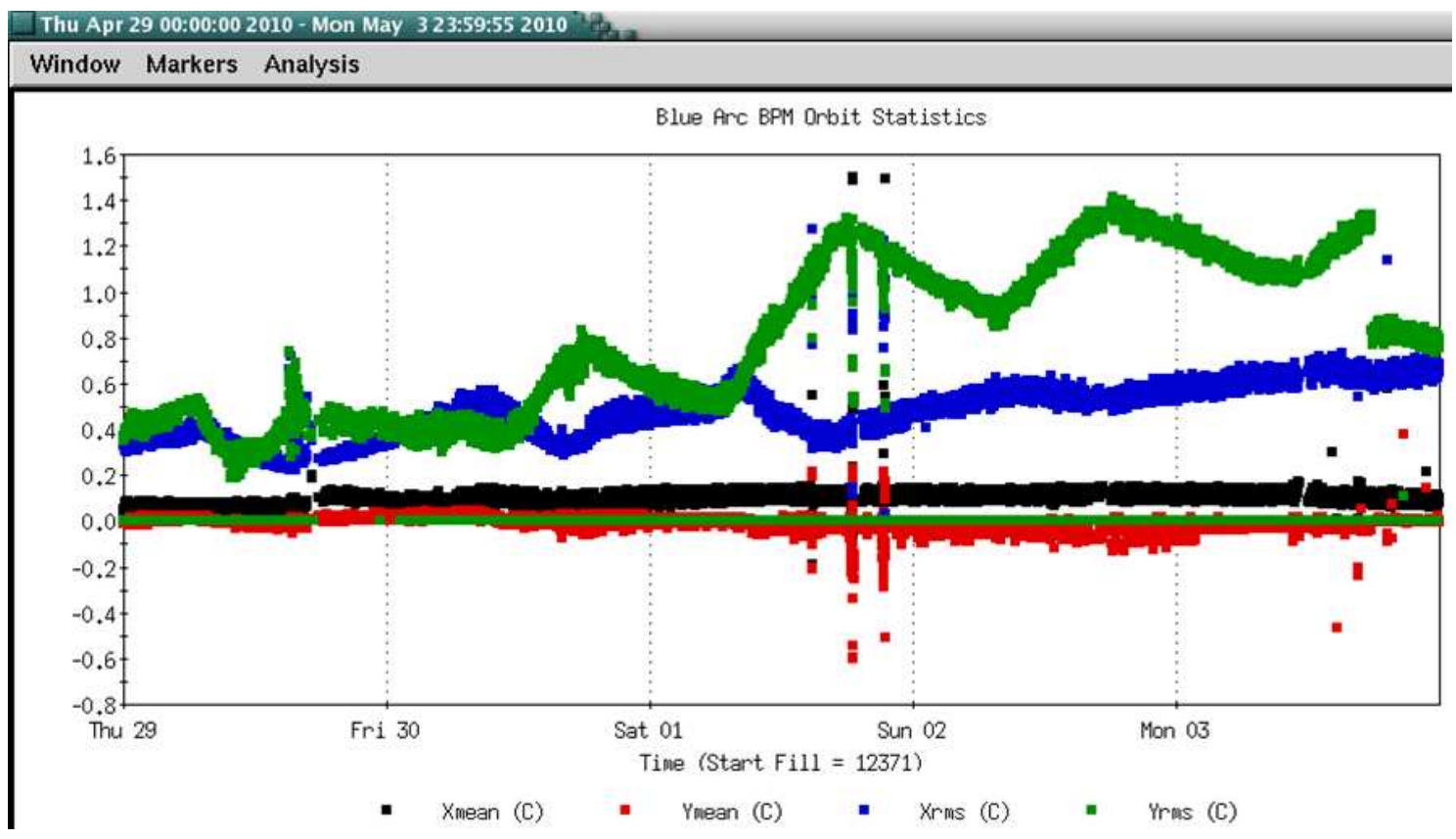

Figure 6: BPM orbit statistics in the arcs of the Blue RHIC ring. The $24 \mathrm{~h}$ variation in the vertical RMS orbit ("Yrms") is clearly visible, together with a much slower drift over several days. 


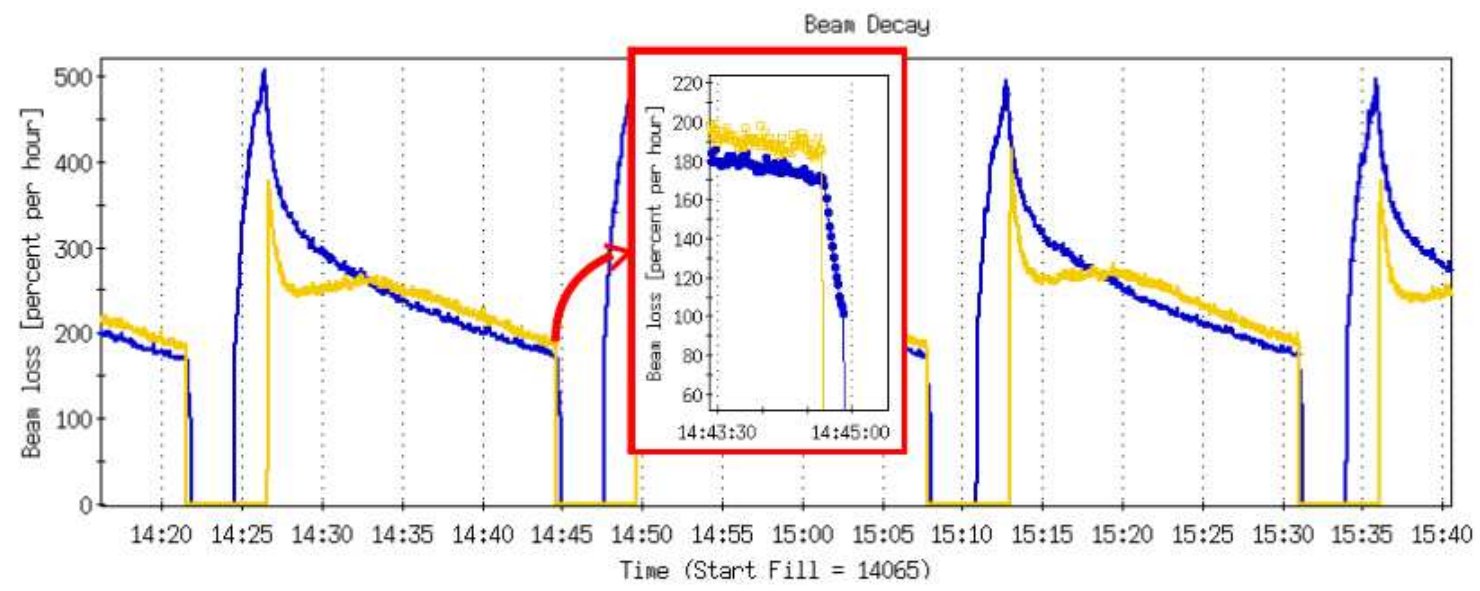

Figure 7: Beam decay rates during several $\sqrt{s_{\mathrm{NN}}}=11.5 \mathrm{GeV}$ stores. At the end of each store, the Blue beam decay dramatically improves as soon as the Yellow beam is dumped (see insert). Note that the algorithm to calculate the beam decay has a time constant of 20 seconds. Hence, the actual drop in instantaneous beam decay is even more dramatic than suggested by this picture.

revealed that the measured $b_{2}$ component of the main dipoles at this energy was not correctly implemented in the online model, leading to a discrepancy between machine and model chromaticity of roughly 100 units [4].

\section{Collimation}

The successful data accumulation in the $\sqrt{s_{\mathrm{NN}}}=7.7 \mathrm{GeV}$ and $11.5 \mathrm{GeV}$ was to a large extend ensured by injecting with the collimators in their inserted position, hence eliminating the need for retracting and re-inserting them at every injection cycle. Since time-consuming re-insertion would have had to happen during the beginning of each store when the luminosity is highest, a significant fraction of the data would have been lost. At both energies collimation was efficient to allow both STAR and PHENIX to run efficiently, while only the primary collimators were used. Figure 9 shows the signals from the individual ZDC units on either side of the STAR detector. Data from the two stores is compared, one without collimation, and one with collimators inserted. Collimation was also required to limit beam losses in uncontrolled areas of the accelerator [5]. However, the collimators needed constant caretaking and retuning after each orbit correction procedure.

\section{Summary}

The RHIC low energy run in FY2010 was successful at both $\sqrt{s_{\mathrm{NN}}}=11.5$ and $7.7 \mathrm{GeV}$. Delivered integrated luminosities met or exceeded the minimum goals at both energies, but improvements may still be possible in future runs. Since no ramps were required between stores, more than 80 percent of the total time was actually spent at store.

Though cold magnet measurements were performed in preparation of the run, the results of these measurements were not used efficiently. With a dedicated hysteresis cycle, sextupole errors $b_{2}$ in the dipoles could be reduced, especially at $\sqrt{s_{\mathrm{NN}}}=7.7 \mathrm{GeV}$ when they are largest. This should in turn result in better beam and luminosity lifetimes due to the reduced nonlinearity of the lattice.

Furthermore, these new magnet multipole data had not been incorporated into the RHIC online 


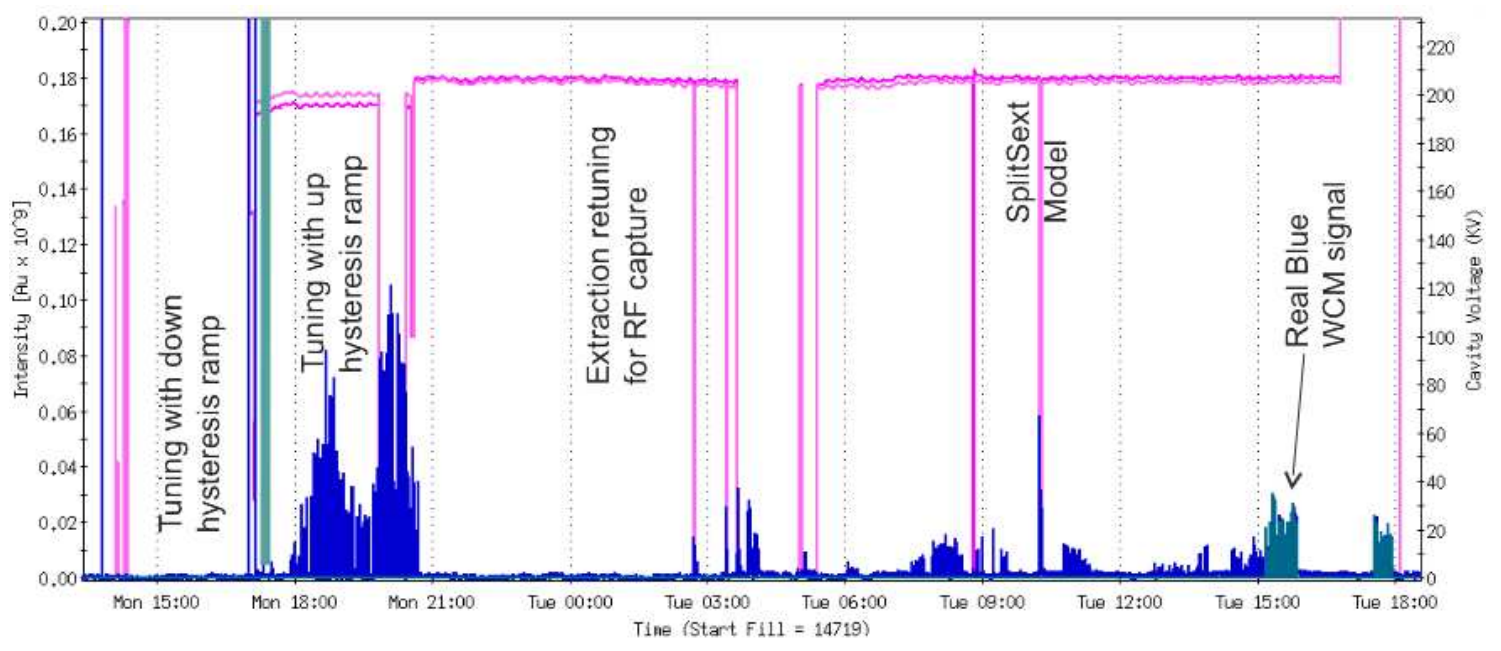

Figure 8: Blue beam currents during the $\sqrt{s_{\mathrm{NN}}}=5 \mathrm{GeV}$ test run.

model. The large resulting chromaticity error of approximately 100 units made beam operation at $\sqrt{s_{\mathrm{NN}}}=5 \mathrm{GeV}$ nearly impossible. For future runs, the $b_{2}$ fit used in the model needs to be extended to properly include the low-field behavior of the magnets.

\section{References}

[1] K.A. Brown et al., "RHIC Performance for FY10 $200 \mathrm{GeV}$ Au+Au Heavy Ion Run", Proc. IPAC'10

[2] K.A. Drees et al., "Medium Energy Heavy Ion Operations at RHIC", Proc. PAC'11

[3] C. Montag, "Mimicking Bipolar Sextupole Power Supplies for Low-energy Operations at RHIC", Proc. PAC 2011, p. 2241.

[4] C. Montag, "Multipole Error Data Analysis for RHIC Low-Energy Operations", C-A/AP/421.

[5] T. Satogata, "RHIC Low Energy Beam Loss Projections", C-A/AP/360. 

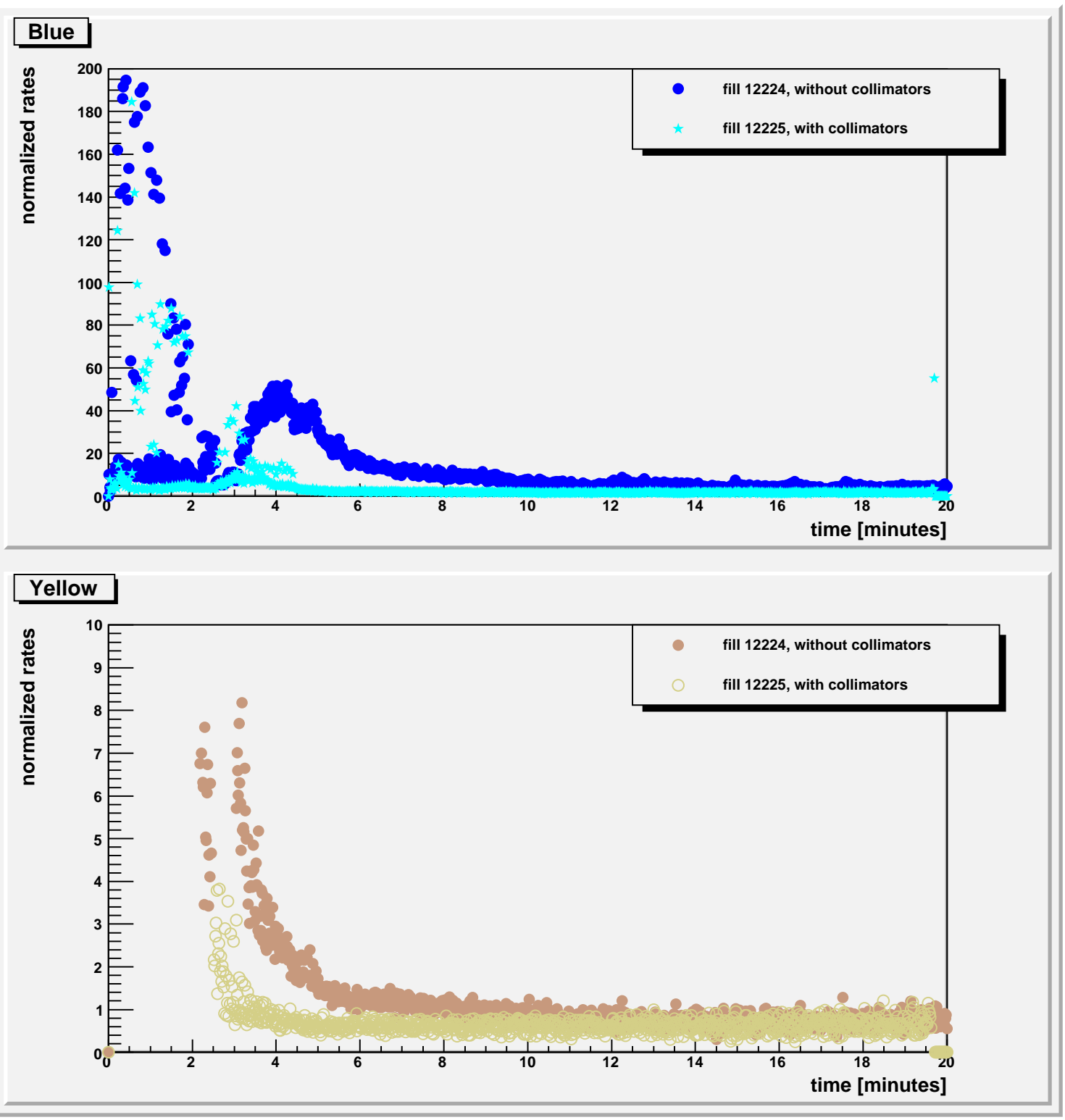

Figure 9: Signals from the individual ZDC units on either side of the STAR detector. Top: Signal from the unit on the side of the incoming blue beam (sector 5). Bottom: Signal from sector 6 . Note the different vertical scales of the two plots. The signal is normalized by the total beam in the machine. The time " 0 " corresponds to the beginning of the injetion process. Yellow is injected about two minutes after blue injection begins. Although the ZDC singles rate is not the most efficient background indicator, collimators prove to reduce the background by at least a factor 3 . 